\title{
Tuberculosis is still a cause of infertility in developing countries
}

\section{P. R. Pant}

Department of Obstetrics and Gynaecology, Tribhuwan University, Teaching Hospital, Institute of Medicine, Kathmandu, Nepal.

Correspondence to: Dr Padam Raj Pant PO Box No12676.Kathmandu, Nepal.

Email: padam2060@yahoo.com

Introduction: Infertility is global problem. Tuberculosis salpingitis is a very rare condition in developed countries but in developing part of the world it is still a problem. One of the commonest symptoms of pelvic tuberculosis is primary infertility. Though infrequent, pelvic tuberculosis is still a cause of infertility in Nepal.

Methods: This is a prospective descriptive study conducted in the 'Infertility clinic', Department of Obstetrics and Gynaecology, Tribhuwan University, Teaching hospital, Kathmandu in 2423 patients from April2005-March2009. All couples were investigated with simple non invasive tests like total blood count with erythrocyte sedimentation rate (ESR), mantoux test, Adenosine diaminase (ADA), polymerase chain reaction (PCR), to invasive procedure like endometrial biopsy to rule out tuberculosis.

Result: Of the total of 2423 infertility among the 37 cases were found to have pelvic tuberculosis contributing to infertility. Of them, 19 patients of them were investigated in private centers for infertility and none of them had investigations for tuberculosis. Out of these 19 cases 5 women were treated in the line of tubal block by hydrotubation without performing investigations for tuberculosis. All of these 37 patients had suspicion of tuberculosis either in the history or investigation. After treatment six patients conceived but only two had successful pregnancy.

Conclusions: Pelvic tuberculosis is still a problem in Nepal affecting the fertility and hence should be considered in the management.

Key words; pelvic tuberculosis, female infertility, tuberculosis, endometritis

\section{Introduction}

Tuberculosis (TB) as a cause of infertility is still a major problem in the developing countries. ${ }^{1}$ Today, though it has become much less common, because of improved socioeconomic conditions, it has been suggested that any women with infertility that cannot be attributed to a specific cause should be investigated for tuberculosis. ${ }^{2}$

Genital TB is always hard to diagnose, because of the fact that it is a silent invader of the genital tract. The only reliable way of making a diagnosis is by actually culturing the tubercle bacillus from tissue sampled from the genital tract. Since it is nearly impossible to take tissues from the fallopian tubes, in practice the diagnosis is usually made by finding tubercle bacilli in the endometrial tissue, obtained by uterine curettage. There are many noninvasive invasive methods for the diagnosis of this condition like endometrial biopsy, Acid Fast Bacilli (AFB) culture, ADA, PCR. ${ }^{3}$

\section{Methods}

A total of 2423 new patients who attended infertility center of TU Teaching Hospital for infertility problem were enrolled in the study after taking verbal consent. These all patients were managed by same doctor for uniformity in management protocol. After detail history taking examination and counseling, couples were investigated starting from simple 
non invasive tests like total blood count with erythrocyte sedimentation rate (ESR), mantoux test, Adenosine diaminase (ADA), polymerase chain reaction (PCR), to invasive procedure like endometrial biopsy to rule out tuberculosis.

\section{Result}

Out of 2423 women, 37 cases were diagnosed as pelvic tuberculosis by either of the method of investigation listed above. Out of these 37 cases 19 patients were treated in private centers before coming to our center. All these 37 patients had history of hysterosalpingogram (HSG) before investigating for tuberculosis. Five of the patients after performing HSG had repeated course of hydrotubation. One of these five patients was attending infertility clinic since 5 years and was subjected to hydrotubation for tubal block. Following hydrotubation she had pain abdomen and evening rise of temperature.

On investigation, she had ascitis on ultrasound, high ESR and ADA positive. Three patients came for second opinion as they were advised tubal surgery as their Hysterosalpingogram was suggestive of bilateral tubal block and on investigations diagnosed tuberculosis

Table 1: Women with infertility and tuberculosis. negative but histopathology suggestive of endometrial tuberculosis. There were altogether 14 cases in which endometrial tuberculosis was confirmed by histopathology including four patients who had AFB culture positive. In Other 23 patient's tuberculosis diagnosis was done by PCR.

Patients attending our center were investigated for tuberculosis by non invasive or invasive methods before doing hysterosalpingogram.

After diagnosis of tuberculosis antituberculosis treatment was started and following treatment six patients conceived but only two had successful pregnancy.

\section{Discussion}

According to a retrospective study in All India Institute of Medical Sciences by $N$ Singh and collogue Genital tuberculosis is a major cause of tubal factor infertility in developing countries. He studied 140 women with infertility and out of them $70(50 \%)$ had tubal factor of infertility. Out of these 70 patients with tubal factor 34 patients $(48.5 \%)$ had tuberculosis. ${ }^{4}$

Patients with pelvic tuberculosis presents with different symptoms. In a retrospective study in India on 94 women it was found that, menstrual abnormalities were seen in only

$\begin{array}{lll} & \text { Number of patients } & \text { Percentage } \\ \text { Total patient with tuberculosis } & 37 & 1.5 \% \\ \text { Positive past history of tuberculosis } & 24 & 64.9 \% \\ \text { menstrual symptoms suggestive of tb } & 11 & 30 \% \\ \text { patients with raised ESR } & 34 & 91.9 \% \\ \text { Patients with positive Mantoux test } & 25 & 69.9 \% \\ \text { AFB positive endometrial sample } & 12 & 30 \% \\ \text { Histological diagnosis in endometrial sample } & 14 & 38 \% \\ \text { Typical beaded appearance in HSG } & 4 & 10 \%\end{array}$

Out of these 37 patients twenty-four patients had history of tuberculosis in the past. Thirteen patients had no any symptoms and signs suggestive of systemic tuberculosis. However 11 of them had typical menstrual history of menorrhagia initially followed by decreased menstrual flow. Thirty-four out of 37 patients had high ESR, three patients had ESR within normal range but diagnosis was histologically proved by endometrial biopsy. Out of 37 patients 25 had positive Mantoux test. 13 patients had already hysterosalpingogram performed 4 of them had typical beaded appearance of the tubes.

Out of 37 patients only in 12 cases pelvic tuberculosis diagnosis was confirmed by Acid fast Bacilli culture in endometrial sample. Seven patients had AFB culture
8 patients: hypo menorrhea and secondary amenorrhea. A diagnostic hysteroscopy showed that 11 had uterine adhesions (18.9\%) and 1 patient had pale endometrium. ${ }^{5}$ According to similar Study by $\mathrm{N}$ Gupta, et al also tuberculosis is common in developing countries including India. So patients seeking infertility should be investigated using methods like clinical and laparoscopic diagnosis, along with endometrial histopathology studies, acid fast bacillus culture, and Polymerase Chain reaction assays, which are considered best available method for diagnosis of tuberculosis in infertility. ${ }^{6}$

Another prospective study done by Mohamed Nezar, et al in Egypt on 420 patients they found 24 cases (5.7\%) pelvic tuberculosis patients diagnosis confirmed by endometrial 
biopsy. According to him genital tuberculosis is the major causative factor for severe tubal disease requiring assisted reproduction in developing countries like India.

In this study there were 37 diagnosed cases of pelvic tuberculosis from the total of 2423 infertility patients Despite of availability of different investigations, most of them were not properly worked up in the line of tuberculosis. ${ }^{7}$ Though the incidence of tuberculosis is only $1.5 \%$, the patient treated for infertility in some centers were not managed properly, missed the investigations in the line of tuberculosis though their history suggestive of some suspicious of tuberculosis.

\section{Conclusions}

Tuberculosis is still a cause of infertility in the developing countries like Nepal; many patients were neglected in performing the investigations in the line of tuberculosis while investigating the infertile women. Tuberculosis investigations, non-invasive or invasive must be done in women seeking infertility treatment in these developing countries not to flare up this pathology further.

\section{References}

1. Mandal SK, Dutta TK. A ten years clinico- pathological study of female genital tract tuberculosis and impact on fertility. J. Nepal Med Assoc. 2009 JanMar;48(173):52-7.

2. Kumar P, Shah NP, Singhal A, Chauhan DS, Katoch VM, Mittal S, et al. Association of tuberculosis endometritis with infertility and other Gynaecological complaints in India. J of Clin Microbiology 2008, Dec 46(12):4068-70.

3. Annamraju H, Ganapathy R, Webb B. Pelvic tuberculosis reactivated by in vitro fertilization egg collection?. Fertil Steril 2003, November; 90(5):1-3.

4. Singh N, Sumana G, Mittal S. Genital tuberculosis: a leading cause for infertility in women seeking assisted conception in Northern India.Archives of Gynecology and Obstetrics 2007 Vol 278; November:325-7.

5. Sharma JB, Roy KK, Pushparaj M, Kumar S. Hysteroscopic findings in women with primary and secondary infertility due to genital tuberculosis. International $\mathrm{J}$ of gynecol and obstet, 2009 Jan;104(1):49-52.

6. N.Gupta, JB Sharma, S Mittal, N Singh, R Mishra, M Kukrela. Genital tuberculosis in infertile
patients.International Journal of Gynecology Obstetrics 2007 Vol 97(2);135-8.

7. Mohamed Nezar, Hosam Goda, Mohamed El-Negery, Miasa El-Saied, Ali A.Wahab, Ahmed M. Badawy. Infertility due to genital tuberculosis. Published online $5^{\text {th }}$ March 2009.

8. Ojo BA, Akaubi AA, Odimayo MS. Endometrial tuberculosis in Nigerian middle belt: a eight years review. Trop Doct. 2008 Jan;38(1):3-4. 